\title{
riginal A rticle
}

\section{Rockall score of the acute upper gastrointestinal bleeding patients the experience in Sudan

\author{
Hussein M Salih ${ }^{1}$, M.A.M Ibnouf ${ }^{2}$, Abdulaziz A Siddig ${ }^{2}$, Abdelmagid M Masaad²
}

\begin{abstract}
Introduction: Innovation of scoring systems helps to rectify personal experience based on subjective evaluation of outcome of patient treatment.

Objectives: To predict the morbidity and mortality in patients presenting with acute upper gastrointestinal bleeding at Ibn-Sina Hospital using the Rockall score.

Patients and methods: Prospective hospital-based study conducted from June 2007 through December 2007 at the Ibn-Sina Hospital Bleeding Centre. Demographic, data of history and physical examination and results of laboratory investigations of 238 patients were collected and allotted a Rockall score, Child-Pugh class and fed to Statistical Package of Social Sciences (SPSS) to calculate means and find the levels of statistical differences and define the predicted and observed mortality rates.

Results: The mean $( \pm \mathrm{SD})$ age $44.6( \pm 15.31)$ range $(8-85)$ years. There were $190(79 \%)$ males. Patients with oesophageal varices, peptic ulcer, and upper GI tumours were 215 (90.3\%), 18 (7.6\%), and $5(2.1 \%)$ respectively.

The mean predicted mortality was $3.8 \%$ while the actual observed mortality $3.8 \%$. The mortality in cases of oesophageal varices was $8(3.4 \%)$, while that of bleeding peptic ulcers was one $(0.4 \%)$.

Conclusion: Rockall score is feasible, accurate, effective system for predicting outcome in patients with upper GI bleeding. The risk factor for mortality are Rockall score $>3$, age $>70$ and rebleeding.
\end{abstract}

Key words: Mortality, morbidity, upper GIT bleeding, Rockall score.

$\mathrm{P}$ rediction of outcome of patients with acute upper GI bleeding remains subjective if it is not based on objective outcome measures. The important factors influencing the outcome of acute upper gastrointestinal haemorrhage have been the focus of research and debate since the 1940s.

The Rockall score ${ }^{1}$ was developed as a simple tool to predict mortality in a large, prospective cohort study of patients with upper GI haemorrhage admitted to hospitals in the United Kingdom in 1993. Subsequent studies in the same setting established the internal validity of the Rockall score and suggested that it might be used to identify patients at high risk for mortality and recurrent bleeding.

1. MOH Surgeon, Halfa Elgadida.

2. Faculty of medicine Omdurman Islamic University.
In this prospective study we used the Rockall score to predict mortality in patients presented with upper GI bleeding to the Mohammed Salih Idris Bleeding Centre affiliated to Ibn-Sina Specialized Hospital.

\section{Objectives}

The objective of this study is to predict morbidity and mortality in Sudanese patients presenting with acute upper gastrointestinal bleeding (GI) in Ibn-Sina Hospital using the Rockall score.

\section{Patients and methods}

This is a prospective, descriptive, hospital based study. It was conducted from June through December 2007 Mohammed Salih Idris Bleeding Centre.

Inclusion criteria: All patients presented with acute upper GI bleeding were included in this study. Informed consent was obtained. Exclusion criteria: Patient who has melaena or haematochezia and the cause 
was not upper GI pathology were excluded from this study. The

Therefore, the studied population consisted of 238 critically ill patients with acute upper GI bleeding.

Set up: The bleeding centre consists of well equipped ICU, three general wards, each with three beds. It has a well equipped endoscopic unit, blood bank, laboratory facilities, and pharmacy. Elective GIT manometry, statistic office, and electronic library are primary available facilities. The centre receives critically ill patients with active upper and lower GI bleeding who were referred from other hospitals.

Clinical and laboratory data conforming to Rockall score and Child-Pugh classification were collected on admission. These include age, co-morbidity factors, mean $\mathrm{BP}$, pulse rate, endoscopic diagnosis and stigmata of recent haemorrhage, presence of ascites, jaundice, total bilirubin, serum albumin, and prothrombin time.

\section{Statistical analysis}

Data were fed to Statistical Package of Social Sciences (SPSS). Descriptive statistics and cross tabulation was performed as appropriate and $\mathrm{P}<0.05$ was taken for statistical significance.

\section{Results}

A total of 238 patients were included in this study. There were 190 (79.8\%) males. The mean $( \pm \mathrm{SD})$ age was $44.6( \pm 15.3)$ range $(8-85)$ years. $190(79.8 \%)$ patients were less than 60 years, $46(19.3 \%)$ have age range from $60-79$ and only $2(0.8 \%)$ patients more than 80 years.

A sum of 58(24.4\%) patients had comorbid diseases and at presentation $41(17.2 \%)$ patients were haemodynamically unstable.

Prophylactic and/or treatment with antibiotics namely Ceftriazxone (Samixon, Elhekma Company) were prescribed for $107(45 \%)$ patients.

The cause of acute upper GI bleeding was oesophageal varices in $215(90.3 \%)$, peptic ulcer in $18(7.6 \%)$, and upper GI tumours in $5(2.1 \%)$ patients.

(c) Sudan JMS Vol. 4, No. 3, Sept 2009
Emergency endoscopy revealed that $114(47.9 \%)$ patients had major stigmata of recent haemorrhage and $124(52.1 \%)$ had blood in the stomach.

The bleeding in $180(75.6 \%)$ patients stopped with $5 \%$ Ethanolamine oleate (EAO) injection, 13(5.5\%) needed histoacryl, $18(7.6 \%)$ adrenaline, one $(0.4 \%)$ with balloon tamponade, one $(0.4 \%)$ with rubber band ligation, $19(8 \%)$ with both EAO $5 \%$ and balloon tamponade while $6(2.5 \%)$ patients were not injected at all and no patient underwent surgery.

Early rebleeding after endoscopic intervention, i.e. in the first 24 hours occurred in $18(7.6 \%)$ patients. $224(94.1 \%)$ patients were discharged home after successful management, five $(2.1 \%)$ patients were transferred to the hospital wards for further treatment, but nine $(3.8 \%)$ patients died.

The Rockall score ranged from 1-10 with mean $( \pm$ SD) $3.5882( \pm 1.83226)$. The predicted mortality ranged from $3.7 \%-5.6 \%$ with mean predicted mortality $3.8 \%$. The actual mortality was $9(3.8 \%)$ patients. Among the deaths eight patients had oesophageal varices and one patient had peptic ulcer. No mortality was seen among patients transferred to the wards. Patients who had Rockall score $>2$ were 166 (69.7\%). All deaths had Rockall score ranged from 1-10 with mean 1.93.

Child Pugh classification was also used as disease specific scoring system so as to find out its prediction of mortality. Child class A patients were $146(61.3 \%)$ with three deaths, which represented $1.3 \%$ of the studied population. Patients with child class B were $65(27.3 \%)$ with only one death, which represented $0.4 \%$ of the studied population. Those with Child class C were 27 (11.3\%) patients with fife deaths, which represented $9.2 \%$ of the studied population.

The mortality in those who had oesophageal varices was $8(3.4 \%)$; and in those who had peptic ulcer was one $(0.4 \%)$. Total mortality was nine $(3.8 \%)$ patients. Six of them were females.

The outcome in those who received antibiotic were as follow $98(91.6 \%)$ patients discharged home, two (1.9\%) transferred, 
seven $(6.5 \%)$ patients died. While the outcome in those who did not receive antibiotic were $126(96.2 \%)$ discharged home, three $(2.3 \%)$ transferred, and two $(1.5 \%)$ died).

The Rockall score in those who receive antibiotic were $30(28 \%)$ patients with score less than two and $77(72 \%)$ with score more than two. In contrast to those who did not receive antibiotic $42(32.1 \%)$ patients had score less than two and $89(67.9 \%)$ had score more than two.

Regarding the outcome in those who re-bled after endoscopy six $(2.5 \%)$ died and $12(5 \%)$ discharged home.

Seven $(3.7 \%)$ out of the 190 patients less than 60 years of age died. Two $(0.8 \%)$ patients their age were more than 80 years died.

The hospital stay for the studied group revealed that those who stayed $<12$ hours were $98(41.2 \%)$ patients but four $(4.1 \%)$ of them died. Those who stayed 13-48 hours were $62(26.1 \%)$ patients with one $(1.6 \%)$ death while those who stayed $>48$ hours were $78(32.8 \%)$ patients with four $(5.1 \%)$ deaths.

\section{Discussion}

In this study the mean age was 44.6 years which is similar what was reported before in Sudan ${ }^{2}$. This is also comparable to that reported in North Ireland where mean age was reported to be 59 years ${ }^{3}$.

In our study, the upper GI bleeding was found to be common in males which similar to the study reported earlier from Sudan ${ }^{4}$. This is most likely explained as by prevalence of oesophageal varices in farmers which are mainly male jobs.

The main cause of acute upper GI bleeding in this study is oesophageal varices; in contrast to the fact that peptic ulcer was the main cause of bleeding in a study done in Germany ${ }^{4}$ and USA $^{1}$ and that is because of bilharzia is an endemic disease in Sudan.

This study showed high association with co-morbid diseases in $24.4 \%$. Similar findings were reported before ${ }^{1}$ reaching $22.2 \%-26.7 \%$.

$17.2 \%$ of the patients were unstable and $44.1 \%$ were haemodynamically stable (C) Sudan JMS Vol. 4, No. 3, Sept 2009 with lower normal BP at presentation making a total number of $61.3 \%$ patients in shock or impending shock.

Major stigmata of recent haemorrhage were seen in $47.9 \%$ patients, while in a study done in Canada ${ }^{5}$ found it $31 \%$ and that may indicate late referral of Sudanese patients. Rebleeding after endoscopy was seen in $7.6 \%$ patients of the study population which is similar to a study done in Italy $^{6}$ that showed rebleeding rate $5.35 \%$.

The mortality rate in this study was $3.8 \%$ which is similar to a study done in $\mathrm{USA}^{5}$ where mortality was reported as $2.1 \%$, while in previous study done in Sudan ${ }^{2}$ reported the mortality as $26.7 \%$. This is probably due to improvement in the facilities of the Bleeding Centre with enough blood and blood products in the blood bank, use of somatostatin analogue for patients with bleeding varices and Injectable PPIs for patients with bleeding ulcers, and the early intervention to stop bleeding.

Antibiotic prophylaxis during acute bleeding episodes in patients with cirrhosis has resulted in significant decreases in mortality rates, but did not become standard protocol until recent years ${ }^{7}$. However, in our study it seems the impact of bleeding override the benefits of prophylactic antibiotics. However, the use of prophylactic antibiotics in bleeding patients with end stage liver disease in Sudan needs further study.

The mean Rockall Score in this study 3.5882 is similar to a study done in $\mathrm{UK}^{8}$ in which the mean Rockall score was 4.

Most dead patients in our study were Child class $\mathrm{C}$ which is similar to a recent study done in North America ${ }^{7}$ that proved the association of the increased mortality with the increased Child score. In our study rebleeding was associated with increased mortality which is similar to a study reported in Germany ${ }^{4}$.

In our study we found the distribution of mortality according to the age group; that in those less than 60 years the mortality is seven $(3.7 \%)$ out of 190 patients in contrast to those more than 80 years the mortality is two out of two, i.e. $100 \%$. So the mortality increased proportionally with age as 
concluded in a study from $\mathrm{UK}^{9}$.

\section{Conclusion}

The mean Rockall Score in this study is 3.5882. Rebleeding after endoscopy is seen in $7.6 \%$. Rebleeding is associated with increased mortality. The mortality rate in this study is $3.8 \%$. Most of the mortality in this study was Child class C. Mortality increased proportionately with age. Death occurred at Rockall score (5.88). Therefore Rockall score is an effective tool for outcome prediction in patients with acute upper GI bleeding.

\section{References}

1- Rockall TA, Logan RF, Devlin HB et al. Risk assessment after acute upper gastrointestinal haemorrhage. Gut 1996; 38; 316-321.

2-Abdelrhim EM, IbnOuf MAM, Adam AM. A Modified APACHE II Score for Predicting Mortality of Variceal Bleeding. Sudan J Medical Sciences 2007; 2: (2); 105-109.

3- Tham TCK, James C, Kelly M. Predicting outcome of acute non-variceal upper gastrointestinal haemorrhage without endoscopy using the Rockall score. Postgraduate Medical J 2006; 82; 757-759.

4- Klebl F, Bregenzer N, Schofer L et al. Risk factors for mortality in sever gastrointestinal bleeding. Int $\mathbf{J}$ Colorectal Dis 2005; 20:49-56.

5- Das A, Ben-Menachem T, Farooq FT et al. Artificial Neural Network as a predictive instrument in patients with acute non-variceal upper gastrointestinal haemorrhage. Gastroenterology 2008; 134; 65-74.

6- Boonpongmanee S, Fleischer DE, Pezzullo JC et al. The frequency of peptic ulcer as a cause of upper-GI bleeding is exaggerated. Gastrointest Endosc. Jun 2004; 59: (7); 788-94.

7- Bambha K, Kim WR, Pedersen R et al. Predictors of early re-bleeding and mortality after acute variceal haemorrhage in patients with cirrhosis. Gut 2008; 57; 814-820.

8- David SS, Mike PJ, Simon JG et al. Effectiveness of an upper gastrointestinal haemorrhage unit: a prospective analysis of 900 consecutive cases using Rockall score as a method of risk standardization. European j of gastroenterol and Hepatol 2004; 16 :( 5); 487-494.

9- Church NI, Dallal HJ, Masson J et al. Validity of the Rockall scoring system after endoscopic therapy for bleeding peptic ulcer: a prospective cohort study. Gastrointestinal endoscopy 2006; 63: (4); 606-612. 